\title{
PSEUDO-COMPLEMENTS IN POSETS 1
}

\author{
P. V. VENKATANARASIMHAN
}

Abstract. In this paper a theory of pseudo-complements is developed for posets (partially ordered sets). The concepts of ideal and semi-ideal are introduced for posets and a few results about them are obtained. These results together with known results about pseudo-complements in distributive lattices lead to the main results. It is proved that if in a pseudo-complemented semilattice or dual semilattice every element is normal, then it is a Boolean algebra. Using this result new proofs for two known theorems are obtained. The existence of maximal ideals in posets is established and it is shown that the dual ideal of dense elements of a poset with 0 is the product of all the maximal dual ideals.

Already, there exists a theory of pseudo-complements for lattices. Frink [5] has obtained a generalisation of the theory for semilattices. In this paper we extend some of the results of Frink [5] and Balachandran [1] to posets (partially ordered sets). We obtain these extensions by using the concept of semi-ideal, which we define in $\$ 2$.

This paper consists of three sections. In $\$ 1$ we summarise some known results which we use in later sections. \$2 deals with some of the properties of semi-ideals and ideals in posets. Our definition of poset ideal is different from that introduced by Frink [4]; however in a lattice our definition is equivalent to the usual definition. Using the results obtained in $\$ 2$, we develop a theory of pseudo-complements for posets in $\S 3$.

1. Preliminaries. We shall denote the ordering relation in a poset by $\leqq$.

Let $A=\left\{a_{i} \mid i \in I\right\}$ be a subset of a poset $P$. Then the least upper bound (l.u.b.) and the greatest lower bound (g.l.b.) of $A$ are also called the lattice-sum and the lattice-product of the $a_{i}$; they are denoted by $\sum_{i \in I} a_{i}$ and $\prod_{i \in I} a_{i}$ respectively. When $A$ is finite, say, $A=\left\{a_{1}, a_{2}, \cdots, a_{n}\right\}$, the lattice-sum and the lattice-product of the $a_{i}$ are denoted by $a_{1}+a_{2}+\cdots+a_{n}$ and $a_{1} \cdot \ldots \cdot a_{n}$ respectively. The

Received by the editors February 3, 1970.

AMS 1969 subject classifications. Primary 0620; Secondary 0630, 0635.

Key words and phrases. Poset, lattice, semilattice, Boolean algebra, ideal, semiideal, complement, relative-complement, pseudo-complement, quasi-complement, normal element, dense element.

1 This paper is a part of the author's Ph.D. thesis prepared under the guidance of Professor V. S. Krishnan and Professor V. K. Balachandran. 
least and greatest elements of a poset, when they exist, are denoted by 0 and 1.

A subset of a lattice $L$ which contains the lattice-sum in $L$ in every pair of its elements is called an additive subsystem of $L$. A multiplicative subsystem is defined in a dual fashion. A subset of a complete lattice $L$ is called a $\Sigma$-subsystem of $L$ if it contains the latticesum in $L$ of any number of its elements. Dually we define a $\Pi$-subsystem. A subset of a complete lattice $L$ which is both a $\Sigma$-subsystem and a $\Pi$-subsystem is called a complete sublattice of $L$.

In a lattice $L$, a sum $\sum_{i \in I} a_{i}$ is said to be distributive if for every $b \in L, b \cdot \sum_{i \in I} a_{i}=\sum_{i \in I} b \cdot a_{i}$. A latttice in which every sum is distributive is called a $\Sigma$-distributive lattice. $\Pi$-distributively is defined dually.

We shall denote the pseudo-complement of an element $a$ in a lattice $L$ by $a^{*}$. The pseudo-complement of $a^{*}$ will be denoted by $a^{* *}$, that of $a^{* *}$ by $a^{* * *}$ and so on.

Lemma 1 [2, p. 49]. If $P$ is a poset with 0 in which every nonvoid subset has a l.u.b. (or dually) then $P$ is a complete lattice.

Lемма 2 [2, p. 148]. In a distributive lattice $L$ closed for pseudocomplements, the following results hold:

(i) $a \leqq a^{* *}$ for every $a \in L$.

(ii) $a \leqq b \Rightarrow a^{*} \geqq b^{*}$ for $a, b \in L$.

(iii) $a^{* * *}=a^{*}$ for every $a \in L$.

(iv) $(a+b)^{*}=a^{*} \cdot b^{*}$ for every pair $a, b \in L$.

(v) $(a \cdot b)^{*}=\left(a^{*}+b^{*}\right)^{* *}$ for every pair $a, b \in L$.

(vi) $(a \cdot b)^{* *}=a^{* *} \cdot b^{* *}$ for every pair $a, b \in L$.

(vii) The set $N$ of normal elements of $L$ forms a Boolean algebra under the same ordering relation as in $L$. Further $N$ is a multiplicative subsystem of $L$ and the lattice-sum $a \oplus b$ of any pair $a, b \in N$ is given by $a \oplus b=(a+b) * *$.

REMARK. By finite induction the results (iv), (v) and (vi) of the above lemma can be extended to any finite number of elements.

LEMMA 3 [3]. A set $S$ is a Boolean algebra if and only if it is closed under a binary operation $(\cdot)$ and a unary operation $\left({ }^{*}\right)$ satisfying the following postulates:

(i) $a \cdot a=a$ for every $a \in S$.

(ii) $a \cdot b=b \cdot a$ for every pair $a, b \in S$.

(iii) $a \cdot(b \cdot c)=(a \cdot b) \cdot c$ for every triple $a, b, c \in S$.

(iv) There exists an element 0 in $S$ such that $a \cdot b^{*}=0 \Leftrightarrow a \cdot b=a$ for $a, b \in S$. 
Lemma 4 [2, p. 147]. A $\Sigma$-distributive complete lattice is closed for pseudo-complements.

2. Semi-ideals and ideals. A nonnull subset $A$ of a poset $P$ is called a semi-ideal if $a \in A, b \leqq a(b \in P) \Rightarrow b \in A$. A nonnull subset $A$ of $P$ is called an ideal if (i) $A$ is a semi-ideal and (ii) the lattice-sum of any finite number of elements of $A$ whenever it exists, belongs to $A$. Dual semi-ideal and dual ideal are defined dually. The set of all elements $x$ of $P$ such that $x \leqq a$ for some fixed $a \in P$ forms an ideal of $P$. It is called the principal ideal generated by $a$ and is denoted by $(a]$. Dually we have the notion of the principal dual ideal $[a)=\{x \mid x \geqq a\}$. By a maximal ideal (maximal dual ideal) of a poset with 0 (1) we mean a maximal element of $I_{\mu}\left(I_{\alpha}\right)$ (for the definitions of $I_{\mu}$ and $I_{\alpha}$ see Theorem 2).

In this paper, set-inclusion, set-union and set-intersection will be denoted by $\subseteq, U$ and $\cap$ respectively.

The following result is easily proved.

THEOREM 1. The set $S_{\mu}$ of all semi-ideals of a poset with 0 is a complete $\Sigma, \Pi$-distributive lattice under $\subseteq$ as ordering relation. If $A$ $=\left\{A_{i} \mid i \in I\right\}$ is any subset of $S_{\mu}, \cup_{i \in I} A_{i}$ and $\bigcap_{i \in I} A_{i}$ are respectively the l.u.b. and the g.l.b. of $A$. Similar result holds for the set $S_{\alpha}$ of all dual semi-ideals of a poset with 1 .

Corollary. $S_{\mu}$ is closed for pseudo-complements and quasi-complements.

The above result follows immediately from Theorem 1, Lemma 4 and its dual.

The psuedo-complement of a semi-ideal $A$ in $S_{\mu}$ is denoted by $A^{*}$. It is easily seen that $A^{*}=\{x \mid(x] \cap(a]=(0]$ for every $a \in A\}$. Since the set-intersection of any family of ideals of a poset $P$ with 0 is an ideal and $P$ itself is an ideal of $P$ the following theorem is clear from Lemma 1.

TheOREM 2. The set $I_{\mu}$ of all ideals of a poset $P$ with 0 is a complete lattice under set-inclusion as ordering relation and $I_{\mu}$ is a multiplicative subsystem of $S_{\mu}$. Similar result holds for the set $I_{\alpha}$ of dual ideals of $a$ poset with 1.

We shall denote the lattice-sums in $I_{\mu}$ by the symbol $\mathrm{V}$.

Lemma 5. In a poset $P$, a lattice-product $\prod_{i \in I} a_{i}$ (lattice-sum $\left.\sum_{i \in I} a_{i}\right)$ exists if and only if $\bigcap_{i \in I}\left(a_{i}\right]\left(\bigcap_{i \in I}\left[a_{i}\right)\right)$ is a principal ideal (principal dual ideal). Also whenever $\prod a_{i}\left(\sum a_{i}\right)$ exists $\cap\left(a_{i}\right]=\left(\prod a_{i}\right]$ $\left(\cap\left[a_{i}\right)=\left[\sum a_{i}\right)\right)$. 
Proof. Suppose $\prod a_{i}$ exists. Then clearly $\left(\prod a_{i}\right] \subseteq \bigcap\left(a_{i}\right]$. Also if $x \in \bigcap\left(a_{i}\right]$, then $x \leqq a_{i}$ for every $i \in I$ and so $x \leqq \prod a_{i}$. Hence $\cap\left(a_{i}\right]$ $\subseteq\left(\prod a_{i}\right]$. It follows that $\cap\left(a_{i}\right]=\left(\prod a_{i}\right]$.

Conversely, if $\cap\left(a_{i}\right]=(x]$, then $x \leqq a_{i}$ for every $i \in I$. Also if $y \leqq a_{i}$ for every $i \in I$, then $y \in \cap\left(a_{i}\right]=(x]$ and consequently $y \leqq x$. It follows that $\prod a_{i}$ exists and $\prod a_{i}=x$. The second part is proved along similar lines.

Lemma 6. In a poset $P$ a finite lattice-sum $a_{1}+a_{2}+\cdots+a_{n}(a$ finite lattice-product $\left.a_{1} \cdot a_{2} \cdot \ldots \cdot a_{n}\right)$ exists if and only if $\left(a_{1}\right] \vee\left(a_{2}\right]$ $\vee \cdots \vee\left(a_{n}\right]\left(\left[a_{1}\right) \vee\left[a_{2}\right) \bigvee \cdots \vee\left[a_{n}\right)\right)$ is a principal ideal (principal dual ideal). Also whenever $a_{1}+a_{2}+\cdots+a_{n}\left(a_{1} \cdot a_{2} \cdot \ldots \cdot a_{n}\right)$ exists $\left(a_{1}\right] \bigvee\left(a_{2}\right] \bigvee \cdots \vee\left(a_{n}\right]=\left(a_{1}+a_{2}+\cdots+a_{n}\right]\left(\left[a_{1}\right) \bigvee\left[a_{2}\right) \bigvee \cdots \vee\left[a_{n}\right)\right.$ $\left.=\left[a_{1}, a_{2} \cdot \ldots \cdot a_{n}\right)\right)$.

Proof. Suppose $a_{1}+a_{2}+\cdots+a_{n}$ exists. Since $a_{1}, a_{2}, \cdots, a_{n}$ $\in\left(a_{1}\right] \vee\left(a_{2}\right] \vee \cdots \vee\left(a_{n}\right], \quad a_{1}+a_{2}+\cdots+a_{n} \in\left(a_{1}\right] \bigvee\left(a_{2}\right] \vee \cdots$ $\vee\left(a_{n}\right]$. Clearly $\left(a_{1}\right] \vee\left(a_{2}\right] \vee \cdots \vee\left(a_{n}\right] \subseteq\left(a_{1}+a_{2}+\cdots+a_{n}\right]$. Hence $\left(a_{1}\right] \bigvee\left(a_{2}\right] \bigvee \cdots \vee\left(a_{n}\right]=\left(a_{1}+a_{2}+\cdots+a_{n}\right]$. Conversely, if $\left(a_{1}\right]$ $\vee\left(a_{2}\right] \vee \cdots \vee\left(a_{n}\right]=(x]$, then clearly $x \geqq a_{1}, a_{2}, \cdots, a_{n}$. Also, if $y \geqq a_{1}, a_{2}, \cdots, a_{n}$, then $y \supseteq\left(a_{1}\right] \bigvee\left(a_{2}\right] \bigvee \cdots \vee\left(a_{n}\right]=(x]$ and so $y \geqq x$. It follows that $a_{1}+a_{2}+\cdots+a_{n}$ exists and $a_{1}+a_{2}+\cdots+a_{n}=x$. The proof of the second part is similar.

Theorem 3. Given an ideal ( $a$ dual ideal) $A$ of a poset $P$ and an element $b \notin A(b \in P)$, among all ideals (dual ideals) containing $A$ and not containing $b$ there exists a maximal one.

PROof. It is easily seen that the set-union of any totally ordered set of ideals (dual ideals) is an ideal (a dual ideal) and is the l.u.b. of the set. Hence the result follows by Zorn's lemma.

Corollary. Any ideal (dual ideal of a poset $P$ with 1(0) is contained in a maximal ideal (maximal dual ideal).

Taking $b=1(b=0)$ in Theorem 3, we get the corollary.

3. Pseudo-complements in posets. An element $a$ of a poset $P$ with 0 is said to have a pseudo-complement $a^{*}$ in $P$ if there exists in $P$ an element $a^{*}$ such that (i) $(a] \cap\left(a^{*}\right]=(0]$ and (ii) for $b \in P,(a] \cap(b]$ $=(0] \Rightarrow(b] \subseteq\left(a^{*}\right]$. It is clear that the pseudo-complement of an element, whenever it exists, is unique. A poset is said to be closed for pseudo-complements if it has 0 and every one of its elements has a pseudo-complement. The concept of quasi-complement is defined in an obvious way using principal dual ideals. An element $a$ of a poset $P$ 
with 0 (not necessarily closed for pseudo-complements) is said to be dense if $a^{*}=0$. Clearly this means $(a] \cap(b]=(0] \Rightarrow(b]=(0]$, for $b \in P$. As in a lattice, an element $a$ of a poset $P$ closed for pseudo-complements is said to be normal if $a=a^{* *}$. The notions of dually dense element and dually normal element are defined in a dual fashion.

REMARK 1. From Lemma 5 it is clear that our definition of pseudocomplement coincides with Frink's definition in a semilattice.

REMARK 2. There exist posets closed for pseudo-complements which are neither lattices nor semilattices. The poset represented by the diagram given below is an example of such a poset.

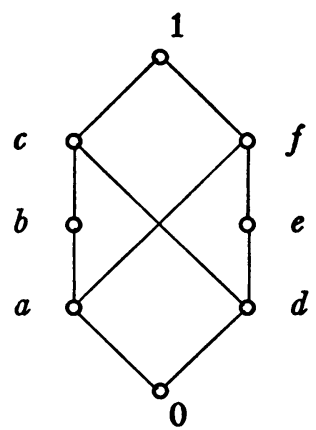

LEMMA 7. In a poset with 0 the pseudo-complement $a^{*}$ of an element $a$ exists if and only if $(a]^{*}$ is a principal ideal. Further whenever $a^{*}$ exists $(a]^{*}=\left(a^{*}\right]$.

Proof. Suppose $a^{*}$ exists. Then by definition $(a] \cap\left(a^{*}\right]=(0]$. Hence $\left(a^{*}\right] \subseteq(a]^{*}$. Now, if $x \in(a]^{*}$, then $(a] \cap(x] \subseteq(a] \cap(a]^{*}=(0)$ and so $(x] \subseteq\left(a^{*}\right]$ by the definition of $a^{*}$. Consequently $(a]^{*} \subseteq\left(a^{*}\right]$. It follows that $(a]^{*}=\left(a^{*}\right]$.

Conversely, if $(a]^{*}=(b]$, then $(a] \cap(b]=(0]$. Also, if $x \in P$ and $(a] \cap(x)=(0]$, then $(x] \subseteq(a]^{*}=(b]$. It follows that $a^{*}$ exists and $a^{*}=b$.

TheOREM 4. In a poset $P$ closed for pseudo-complements, the following results hold:

(i) $a \leqq a^{* *}$ for every $a \in P$.

(ii) $a \leqq b \Rightarrow a^{*} \geqq b^{*}$ for $a, b \in P$.

(iii) $a^{* * *}=a^{*}$ for every $a \in P$.

(iv) $P$ has a greatest element 1 and $1=0 *$.

Proof. (i) Since $a^{* *}$ is the pseudo-complement of $a^{*}$ and $\left(a^{*}\right]$ $\cap\left(a^{* *}\right]=(0]=\left(a^{*}\right] \cap(a]$ the result follows from the definition of $a^{* *}$. 
(ii) $a \leqq b \Rightarrow(a] \subseteq(b] \Rightarrow(a]^{*} \supseteq(b]^{*}$ (applying (ii) of Theorem 2 to $S_{\mu}$ ). Hence by Lemma $7, a \leqq b \Rightarrow a^{*} \geqq b^{*}$.

(iii) Replacing $a$ by $a^{*}$ in (i) we get $a^{*} \leqq a^{* *}$. Also as $a \leqq a^{* *}$ by (i) $a^{*} \geqq a^{* * *}$ by (ii). Hence (iii).

(iv) If $x$ is any element of $P$, clearly $(0] \cap(x]=(0]$ and so by the definition of $0^{*},(x] \subseteq(0]^{*}=\left(0^{*}\right]$. Hence $x \leqq 0^{*}$ so that $0^{*}=1$.

THEOREM 5. In a poset closed for pseudo-complements the following results hold:

(i) If a finite product $a_{1} \cdot a_{2} \cdot \ldots \cdot a_{n}$ exists in $P$, then so does the product $a_{1}^{* *} \cdot a_{2}^{* *} \cdot \ldots \cdot a_{n}^{* *}$. Further

$$
\begin{aligned}
& \left(a_{1} \cdot a_{2} \cdot \ldots \cdot a_{n}\right)^{* *}=a_{1}^{* *} \cdot a_{2}^{* *} \cdot \ldots \cdot a_{n}^{* *} \\
& \quad \text { and }\left(a_{1} \cdot a_{2} \cdot \ldots \cdot a_{n}\right)^{*}=\left(a_{1}^{* *} \cdot a_{2}^{* *} \cdot \ldots \cdot a_{n}^{* *}\right)^{*} .
\end{aligned}
$$

(ii) If a sum $\sum_{i \in I} a_{i}$ exists in $P$ then the product $\prod_{i \in I} a_{i}^{*}$ exists in $P$ and $\left(\sum a_{i}\right)^{*}=\prod a_{i}^{*}$.

Proof. (i) By Lemma 7,

$\left(\left(a_{1} \cdot a_{2} \cdot \ldots \cdot a_{n}\right)^{* *}\right]=\left(a_{1} \cdot a_{2} \cdot \ldots \cdot a_{n}\right]^{* *}=\left(\left(a_{1}\right] \cap\left(a_{2}\right] \cap \cdots \cap\left(a_{n}\right]\right)^{* *}$

(by Lemma 5)

$$
=\left(a_{1}\right]^{* *} \cap\left(a_{2}\right]^{* *} \cap \cdots \cap\left(a_{n}\right]^{* *}
$$

(by (vi) of Lemma 2 applied to $S_{\mu}$ and the remark under Lemma 2)

$$
=\left(a_{1}^{* *}\right] \cap\left(a_{2}^{* *}\right] \cap \cdots \cap\left(a_{n}^{* *}\right]
$$

(by Lemma 7). By Lemma 5 it follows that $a_{1}^{* *}, a_{2}^{* *} \cdot \ldots \cdot a_{n}^{* *}$ exists and $\left(a_{1} \cdot a_{2} \cdot \ldots \cdot a_{n}\right)^{* *}=a_{1}^{* *} \cdot a_{2}^{* *} \ldots . \cdot a_{n}^{* *}$. Hence by (iii) of Theorem 4,

$$
\left(a_{1} \cdot a_{2} \cdot \ldots \cdot a_{n}\right)^{*}=\left(a_{1}^{* *} \cdot a_{2}^{* *} \cdot \ldots \cdot a_{n}^{* *}\right)^{*} .
$$

(ii) Now $\sum a_{i} \geqq a_{i}$ for every $i \in I$ and so by (ii) of Theorem 4, $\left(\sum a_{i}\right)^{*} \leqq a_{i}^{*}$ for every $i \in I$. Also if $x \in P$ and $x \leqq a_{i}^{*}$ for every $i \in I$, then $a_{i} \leqq a_{i}^{* *} \leqq x^{*}$ by (i) and (ii) of Theorem 4 and so $\sum a_{i} \leqq x^{*}$. Hence by another application of (i) and (ii) of Theorem 4 , we get $x \leqq x^{* *} \leqq\left(\sum a_{i}\right)^{*}$. It follows that $\prod a_{i}^{*}$ exists and $\prod a_{i}^{*}=\left(\sum a_{i}\right)^{*}$.

THEOREM 6. Let $P$ be a poset closed for pseudo-complements. If a subset $N$ of normal elements of $P$ is closed for pseudo-complements, contains 0 or 1 and is a semilattice or a dual semilattice, then $N$ is a Boolean algebra. 
Proof. Let $N$ be a semilattice satisfying the other conditions. In view of Lemma 3 , it suffices to prove that $a \cdot b^{*}=0 \Leftrightarrow a \cdot b=a$ for $a, b \in N$. Now if $a \cdot b^{*}=0$, then $a \leqq b^{* *}=b$ as $b \in N$, and so $a \cdot b=a$. On the other hand, if $a \cdot b=a$, then $a \cdot b^{*}=a \cdot b \cdot b^{*}=a \cdot 0=0$. Thus $a \cdot b^{*}$ $=0 \Leftrightarrow a \cdot b=a$.

Now suppose $N$ is a dual semilattice satisfying the other conditions. Define $a \cdot b=\left(a^{*}+b^{*}\right)^{*}$, + denoting the lattice-sum in $N$. Then as $N$ is closed for (*) and (+), $a \cdot b \in N$. Also $a=a^{* *} \geqq\left(a^{*}+b^{*}\right)^{*}$ by (ii) of Theorem 4 as $a^{*} \leqq a^{*}+b^{*}$; that is $a \geqq a \cdot b$. Similarly $b \geqq a \cdot b$. If $x \in N$ and $x \leqq a, b$, then by (ii) of Theorem $4, x^{*} \geqq a^{*}, b^{*}$ and so $x^{*} \geqq a^{*}+b^{*}$. Consequently as $x \in N$, by (ii) of Theorem $4, x=x^{* *} \leqq\left(a^{*}+b^{*}\right)^{*}$ $=a \cdot b$. Thus it follows that $a \cdot b$ is the lattice-product of $a, b$ in $N$. Hence the result follows by the first part.

REMARK. The above theorem is a generalisation of the following theorem of Frink [5]: The normal elements of a semilattice closed for pseudo-complements form a Boolean algebra.

COROLlARY. If in a semilatice or a dual semilattice $S$ closed for pseudo-complements, every element is normal, then $S$ is a Boolean algebra.

The following theorem is due to Varlet [7], but the proof given here is different from his proof.

THEOREM 7. A uniquely complemented lattice $L$ which is also closed for pseudo-complements is a Boolean algebra.

Proof. Let $a \in L$ and $a^{\prime}$ the unique complement of $a$. Then $a \cdot a^{\prime}=0$ and so $a^{\prime} \leqq a^{*}$ where $a^{*}$ is the pseudo-complement of $a$ in $L$. Hence $a+a^{*}=1$. Similarly $a^{*}+a^{* *}=1$. Also $a \cdot a^{*}=0=a^{*} \cdot a^{* *}$. Thus $a$ and $a^{* *}$ are complements of $a^{*}$, and so, as $L$ is uniquely complemented $a=a^{* *}$. Now the result follows by the corollary to Theorem 6 .

We give below a new proof of a known theorem (cf. [6]).

THEOREM 8. A uniquely complemented lattice $L$ which is also relatively complemented is a Boolean algebra.

Proof. Let $a \in L$ and $b$ an element of $L$ such that $a \cdot b=0$. Let $c$ be a complement of $a+b$ in $[b, 1]$. Clearly $a \leqq a+b$ and $b \leqq c$. Hence $a \cdot c=a \cdot(a+b) \cdot c=a \cdot b=0$ and $a+c=a+b+c=1$. Thus as $L$ is uniquely complemented, $c$ is the unique complement of $a$ and so it is independent of $b$; that is, if $x$ is any element such that $a \cdot x=0$ then $x \leqq c$. Hence $c=a^{*}$. Thus $L$ is closed for pseudo-complements. Now the result follows by Theorem 7 . 
Lemma 8. If $\left\{A_{i} \mid i \in I\right\}$ is a family of ideals of a poset $P$ then

$$
\bigvee_{i \in I} A_{i}=\left\{x \mid(x] \subseteq\left(a_{i 1}\right] \vee\left(a_{i 2}\right] \vee \cdots \vee\left(a_{i n}\right], \quad a_{i j} \in A_{i j}\right\}
$$

A similar result holds for dual ideals.

The proof of the above lemma is similar to that of the corresponding result for lattices.

The following theorem generalises the corresponding result for lattices, due to Balachandran [1].

THEOREM 9. The set $D$ of all dense elements of a poset $P$ with 0 is a dual ideal of $P$. Further $D$ is the product of all the maximal dual ideals of $P$.

Proof. For proving this, first we observe that $x \in D \Leftrightarrow(x]^{*}=(0]$.

Now suppose $x \in D, x \notin \cap M_{i}, M_{i}$ being the maximal dual ideals of $P$. Then $x \notin M$, for some maximal ideal $M$ of $P$. Hence $M \vee[x]=P$ and so by Lemma 8 , there exist elements $a_{1}, a_{2}, \cdots, a_{n} \in M$ such that $[0) \subseteq[x) \bigvee\left[a_{1}\right) \bigvee\left[a_{2}\right) \vee \cdots \vee\left[a_{n}\right)$. By Lemma 6 , it follows that $x \cdot a_{1} \cdot a_{2} \cdot \ldots \cdot a_{n}=0$. Consequently $\left(a_{1}\right] \bigvee\left(a_{2}\right] \vee \ldots \vee\left(a_{n}\right] \subseteq(x]^{*}$ $=(0]$ as $x \in D$. Hence by Lemma $5, a_{1} \cdot a_{2} \cdot \ldots \cdot a_{n}=0$ which is absurd as $a_{1} \cdot a_{2} \cdot \ldots \cdot a_{n} \in M \neq P$. It follows that $x \in \cap M_{i}$ and so

$$
D \subseteq \cap M_{i} \text {. }
$$

On the other hand, if $x \notin D$ then $(x]^{*} \neq(0]$ and so there exists an element $y(\neq 0)$ in $P$ such that $(x] \cap(y]=(0]$. By Lemma $5, x \cdot y=0$. Since $y \neq 0$, by the corollary to Theorem 3 , there exists a maximal dual ideal $M$ such that $y \in M$. As $x \cdot y=0, x \notin M$. It follows that $x \notin \cap M_{i}$ and so

$$
\cap M_{i} \subseteq D .
$$

The second part of the theorem follows from (1) and (2). Since the intersection of any family of dual ideals is a dual ideal, the first part follows from the second.

\section{REFERENCES}

1. V. K. Balachandran, $A$ characterisation for complete Boolean algebras, J. Madras Univ. Sect. B 24 (1954), 273-278. MR 16, 439.

2. G. Birkhoff, Lattice theory, 2nd rev. ed., Amer. Math. Soc. Colloq. Publ., vol. 25, Amer. Math. Soc., Providence, R. I., 1948. MR 10, 673.

3. O. Frink, Representation of Boolean algebras, Bull. Amer. Math. Soc. 47 (1941), 755-756. MR 3, 100.

4. - Ideals in partially ordered sets, Amer. Math. Monthly 61 (1954), 223234. MR 15, 848. 
5. - Pseudo-complements in semi-lattices, Duke Math. J. 29 (1962), 505-514. MR 25 \#3869.

6. G. Szász, On complemented lattices, Acta Sci. Math. (Szeged) 19 (1958), 77-81. MR 20 \#2293.

7. J. Varlet, Contribution a l'étude des treillis pseudo-complémentés et des treillis de Stone, Mém. Soc. Roy. Sci. Liège. Coll. in-8 (1963), no. 4, 71 pp. MR 29 \#2200.

University of Kerala, Kariavattom, Trivandrum, India 